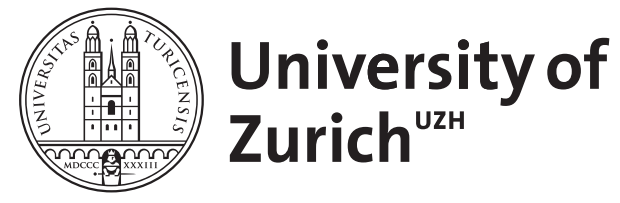

Zurich Open Repository and Archive

University of Zurich

University Library

Strickhofstrasse 39

CH-8057 Zurich

www.zora.uzh.ch

Year: 2003

\title{
La tenson avec le Dieu d'Amour de Monte Andrea
}

\author{
Bartuschat, J
}

Other titles: Les voix multiples. Du conflit au dialogue

Posted at the Zurich Open Repository and Archive, University of Zurich

ZORA URL: https://doi.org/10.5167/uzh-138399

Journal Article

Published Version

Originally published at:

Bartuschat, J (2003). La tenson avec le Dieu d'Amour de Monte Andrea. Arzanà. Cahier de litteràture médiévale italienne, (9):77-91. 


\section{La tenson avec le Dieu d'Amour de Monte Andrea} Johannes Bartuschat

\section{Citer ce document / Cite this document :}

Bartuschat Johannes. La tenson avec le Dieu d'Amour de Monte Andrea. In: Arzanà 9, 2003. Les voix multiples. Du conflit au dialogue. pp. 77-91;

doi : 10.3406/arzan.2003.921

http://www.persee.fr/doc/arzan_1243-3616_2003_num_9_1_921

Document généré le 27/04/2017 


\section{LA TENSON AVEC LE DIEU D'AMOUR DE MONTE ANDREA}

$\mathrm{D}$ ans la très large production de tensons, on peut distinguer, sans prejudice pour des typologies plus fines, deux sortes de dialogue : la tenson, que l'on pourrait appeler réelle, entre deux ou plusieurs poètes, et la tenson fictive, par exemple entre un amant et sa dame'. Comme l'a rappelé Claude Perrus, on peut distinguer, dans les tensons fictives, selon un critère purement formel, les dialogues contenus à l'intérieur d'un même texte (un sonnet ou une chanson, mais aussi une ballade), et ceux qui distribuent les différentes voix sur des poésies séparées (des sonnets notamment).

Le texte de Monte Andrea que nous présentons ici ${ }^{2}$, est original à ces deux titres. Il appartient à la catégorie de la tenson fictive, mais d'un genre particulier puisqu'il s'agit $\mathrm{d}^{\prime}$ une tenson entre le poète (amante) et le Dieu d'Amour (Amore). Cette tenson se compose de douze sonnets (de 16 vers chacun, selon une mode métrique particulierement en vogue chez les poètes guittoniens), soit six sonnets pour chaque interlocuteur en alternance. Cette structure, unique à notre connaissance quant à sa longueur, rappelle le genre métrique de la "couronne de sonnets " qui connaît une production illustre en ces années et dans les décennies suivantes (Chiaro Davanzati, Folgore da San Gimignano); on peut également la rapprocher de tentatives contemporaines de regrouper des sonnets dans des ensembles plus larges, comme c'est le cas chez Guittone 
ou dans la Corona di casistica amorosa. Mais le texte de Monte se distingue de tous ces exemples par sa structure dialogique?

Présentons d'abord rapidement le contenu de cette tenson dont on trouvera ci-après traduits les deux premiers sonnets (nous numérotons les sonnets de 1 à 12).

L'amant se dit désespéré car il ne peut montrer ouvertement sa peine et son amour à sa Dame, et il demande à Amour d'intervenir auprès d'elle en sa faveur (1). Amour refuse et accuse le poète de ne pas être un amant exemplaire; c'est par sa propre faute qu'il souffre, car le vrai service d'amour ne procure que des joies (2). L'amant proteste ct affirme qu'il est parfaitement loyal, et accuse Amour de ne pas récompenser sa fidélité (3). Face à ces protestations, Amour lui adresse un reproche plus concret: le poète a trahi le secret de l'amour (4). L'amant se justifie: sa passion est si violente qu'il ne peut pas faire autrement (5). Amour lui rétorque: si ton amour est aussi grand que tu le prétends, pourquoi ne te satisfais-tu pas d'un amour caché ? (6) Mais l'amant ne relève pas le reproche et revient à la charge: $c^{\prime}$ est par la faute d'Amour qu'il est dans un état proche de la folie; il demande donc a Amour de soumettre la dame, elle aussi, à son joug (7). Amour l'invite à la modération et à la patience: le bon amant réussit à cacher ses sentiments et se satisfait des joies que l'amour lui procure (8). L'amant déclare se soumettre totalement à la volonté d'Amour; il se dit prêt à accepter toutes les souffrances et les punitions, mais implore Amour de ne pas le priver de son conseil (9). En guise de réponse Amour lui adresse un enseignement sur la conduite du parfait amant, qui reste très général: l'amant doit être umile, cortese, largo, gentile et préserver le secret de son amour (10). L'amant déclare qu'il sera le 
fidèle serviteur d'Amour et suivra ses préceptes mais il réitere aussi sa demande d'une récompense (11). Amour lui répond: si tu suis mes enseignements, tu n'auras pas de déplaisir, si tu es un bon amant, tu connaîtras la joie avec ta Dame (12).

Avant d'aborder l'analyse de ce dialogue, il nous faut nous interroger sur l'invention même d'un dialogue entre le poète et Amour, invention, nous l'avons dit, plutôt originale. Si dans la poésie provençale le Dieu d'Amour apparaît fréquemment", les poètes se bornent en général à en invoquer le nom ou à s'adresser à lui pour dire leur peine ou implorer son secours. Mais Amour ne prend presque jamais la parole. En effet, à notre connaissance, on ne recense que trois exemples provençaux de dialogues entre l'amant et Amour. Dans une chanson de Peirol', Amour reproche à l'amant de delaisser son service; l'amant lui demande de ne plus le harceler puisque ce service n'a porté aucun fruit; quand Amour lui rappelle son amour pour sa Dame, le poète lui annonce son départ pour la croisade; Amour lui rétorque que même les rois se dérobent à leur devoir de croisé et qu'il ferait donc bien de rester. Dans une chanson de Raimon Jordan ${ }^{6}$, Amour reproche à l'amant d'avoir abandonné le service d'amour; l'amant lui répond qu'il n'a connu que des souffrances et des déceptions et avoue sa lassitude. Il accuse Amour de lui refuser une récompense à laquelle il aurait pourtant droit, comme serviteur fidele. Amour esquive le reproche, et appelle l'amant à persévérer dans la voie de l'amour en lui promettant les faveurs de la Dame. La chanson Donna per vos estauc en greu turmen d'Aimeric de Peguilhan' se compose de cinq strophes: les trois premières sont consacrées au thème plus traditionnel d'une tenson avec la Dame, les deux dernières contiennent un dialogue avec le 
Dieu d'Amour; le poète y accuse Amour d'indifférence; Amour récuse le reproche et l'invite à la patience. Ce deuxième échange intervient après l'échec du dialogue avec la Dame puisque cette dernière a fait preuve d'une dureté impitoyable, ce qui correspond partiellement à la situation initiale de notre tenson : ne pouvant s'adresser à la Dame, le poète interpelle le Dieu d'Amour.

Bien que peu nombreux, ces précédents nous paraissent significatifs. Dans ces trois textes, le dialogue avec Amour traduit un moment de crise du rapport amoureux, et plus largement de la conception de l'amour (cet amour que nous appelons " courtois"). Le poète las et désespéré est sur le point d'abandonner sa Dame et doute du sens même du service d'amour. Amour gouverne la vie de l'amant en seigneur féodal; ses interventions sont autant de "rappels a l'ordre " face à des amants réticents ou las; si le Dieu d'Amour se défend du reproche d'indifférence, il évite de répondre aux plaintes des amants et à leur demande d'une récompense. Ces observations nous semblent s'appliquer aussi, pour une large part, à la tenson de Monte Andrea et nous fournissent une hypothèse de lecture. Mais examinons d'abord rapidement la forme dialogique de notre texte.

Par rapport au dialogue contenu à l'interieur d'une même unité textuelle qui privilégie l'échange rapide de phrases brèves (voir les textes analysés par Claude Perrus), la couronne de sonnets favorise naturellement l'amplification; or nous pouvons constater que cette amplification est, dans notre tenson, plus rhétorique qu'argumentative. On ne perçoit guère de développement discursif à l'intérieur de l'ensemble; autrement dit, ce dialogue n'a pas les caractéristiques d'un débat, à la différence d'un bon nombre de tensons réelles, notamment dans la poésie italienne. Notre tenson reste fidele aux 


\section{LA TENSON AVEC le Dieu d' AMOUR}

modalités des tensons fictives entre l'amant et la Dame: elle privilégie les actes langagiers tels que la protestation, la lamentation et la supplication (pour l'amant), l'accusation, la promesse (pour Amour). Cette dynamique communicative est soutenue par un apparat rhétorique des plus élaborés.

Illustrons rapidement notre propos par un exemple. Dans le sonnet 1 on ne compte pas moins de onze occurrences du pronom personnel «je » dans ses différentes formes, ce qui souligne bien la fonction expressive, au sens jakobsonien, du texte. Le sonnet 2 (réponse d'Amour) présente l'effet spéculaire, puisque nous y trouvons de nombreuses occurrences du pronom de deuxieme personne (tuo, ti, etc.) qui se prolongent dans une chaîne $d$ 'assonances du son « $t$ » qui lient entre eux les mots-cles du sonnet sur le plan thématique et doctrinal (tormento, dritto, condotto). En outre, Amour propose un nouveau mot-clé, folle, qui apparaît deux fois et qui est suivi par une autre chaîne allitérative (et dérivationnelle) fallire, falso, falli. La requête téméraire et inconsciente de l'amant (folle) est ainsi dénoncée comme expression d'un esprit marqué par ses manquements au code de l'amour; l'amant vit dans l'erreur et a besoin d'être éclairé par Amour pour retrouver le droit chemin. Dans le sonnet 3, l'amant proteste de sa soumission totale à Amour: cette déclaration d'allégeance passe par un réseau très dense de pronoms personnels de première personne (sept occurrences) et de deuxième personne (six occurrences). L'amant répond à la chaîne falli, falso, falli: " giamai non ti feci fallisgione "; mais il reprend surtout l'allitération en $t:$ "Or como trovo in te tanta menzone/che, di me, dicer puo', in tutto: 'Mio!'». La chaîne allitérative continue ("sono, Amor, ver'te tutto leale ») pour s'achever, au dernier vers, sur: "mia vita è mortale ". La faute en revient à la Dame qu'il accuse de le 
dédaigner (« disdegna »). Amour, dans sa réplique, s'en inspire : il repond a l'oxymore «vie-mort " par une invention typiquement guittonienne: la négation au moyen d'une adjonction de préfixe non lexicalisee: "'Amante', no, ma 'disamante'dico " (qui rime avec disdico) ". Cet échantillon peut suffire à illustrer la technique poétique de cet échange: un tissu complexe, dans la tradition de Guittone d'Arezzo, d'échos verbaux, de jeux de sons, de reprises grammaticales, de rimes dérivées, etc. Monte adapte ce style au genre de la tenson puisque ces éléments se répondent d'un sonnet à l'autre et assurent la cohérence de l'ensemble. Notre texte se présente donc comme une véritable joute verbale dans laquelle les deux protagonistes rivalisent d'habileté rhétorique.

La dynamique de l'échange ne repose pas, nous l'avons dit, sur un développement argumentatif ou doctrinal, mais sur l'évolution des rôles respectifs des interlocuteurs. C'est l'amant qui prend l'initiative du dialogue. Cela nous conduirait naturellement à lire l'ensemble comme six paires de sonnets: la proposition de l'amant et réponse d'Amour. En réalité, dès le sonnet 2 Amour renverse la direction de l'échange: il répond à la requête de l'amant par un reproche; dorénavant c'est l'amant qui répondra à Amour pour se justifier. Ce sont les répétitions de mots qui nous révèlent la direction de l'échange: les sonnets de l'amant reprennent systématiquement un mot du sonnet précédent d'Amour, le plus souvent au même vers $(2,7$ : fallire, 3, 7 : fallisgione; 6,1 : scusa, 7,1 : scusa; 6 , 2 : folle, 7, 2 follia; 8,1 : certo, 9, 2 certo; $10,1:$ io so per fermo, 111 : ben saccio; l'absence de cet effet d'écho dans le couple de sonnets 4-5 s'explique sans doute par le fait que l'amant se justifie d'avoir trahi le secret, et que son discours feint, dans une logique de dénégation, d'ignorer celui d'Amour). 
L'amant commence par accuser le Dieu d'Amour et exige son intervention; ensuite il se justifie face aux reproches que lui adresse Amour et finit par implorer sa bienveillance et se mettre sous sa protection. Amour réaffirme ainsi, dans cet échange, son pouvoir et son autorité, dans un sens tout à fait féodal : l'amant doit à son seigneur fidélité et obéissance.

Chez Peirol et Raimon Jordan, c'est Amour qui apostrophe l'amant pour le "rappeler à l'ordre ". Monte Andrea en revanche reprend le motif, topique dans la poésie lyrique, de la plainte adressée au Dieu d'Amour, qu'il développe dans un sens dialogique. Mais remarquons que l'amant s'adresse à Amour avec une attitude ambiguë: pour obtenir sa protection et lui demander son aide, mais aussi pour réclamer son $\mathrm{d} a$, la récompense de son service. S'adresser au Dieu d'Amour comme à un "seigneur " revient à dédoubler la métaphore féodale constitutive de la lyrique d'amour (l'amant vassal qui est au service de sa Dame et espère une récompense pour sa fidélité et sa loyauté); le poète se dit au service d'Amour et non pas seulement de la Dame'. Le poète-amant espère ainsi dépasser l'impasse du service d'amour par une nouvelle logique féodale: fidèle serviteur du seigneur Amour, il peut réclamer sa récompense. En même temps, son désir d'obtenir la protection d'Amour rappelle le motif du Dieu d'Amour comme guide qui console et protège l'amant (amplement present dans la Vita Nuova, mais aussi développé, de façon originale, dans un sonnet de Paolo Lanfranchi ${ }^{\text {(1)}}$ ). L'amant demande donc à Amour d'intervenir auprès de sa Dame; mais il le supplie également de ne pas le priver de son conseil, indispensable pour affronter une passion aussi violente. Amour refuse d'accéder à la requête de l'amant, mais aussi de recevoir ses plaintes; il lui reproche son attitude et ses manque- 
ments. Quant aux conseils, il se borne à lui opposer ses lois: si l'amant les respecte, il connaîtra la joie.

Malgré ses protestations et ses revendications, l'amant doit se satisfaire de cette réponse. A deux reprises, il insiste et réclame sa récompense (encore dans son dernier sonnet: 11, v. 11-16). Mais comme on ne peut contraindre la Dame, on ne saurait contraindre le seigneur, le Dieu d'Amour. La dernière réponse de celui-ci reste vague: il lui promet qu'il connaîtra la joie. Mais quelle est précisément cette joie? Est-ce le joi des troubadours? est-ce l'accomplissement du désir? Amour refuse de lever l'ambiguïté, cette ambiguïté qui est le paradoxe même de l'amour courtois: le service d'amour apparaît à la fois comme le chemin vers l'union avec la Dame, et comme une fin en soi qui, pour se réaliser, doit renoncer à la récompense.

Amour exige la loyauté et le respect de ses lois; c'est à cette condition qu'il propose même, par une formule curieuse, à l'amant un pacte de fidélité réciproque: pour récuser le reproche d'avoir trahi l'amant, il dit s'être livré à lui comme à un ami $\left(4, v .2-3^{11}\right)$, ce qui est le renversement d'un topos du langage amoureux (que l'amant luimême utilise plusieurs fois dans cette tenson). Mais cette figure d'un Dieu d'Amour protecteur et ami, que nous avons entrevue dans les requêtes de l'amant, reste une chimere. Amour se borne à rejeter les plaintes de l'amant comme contraires à ses lois, et a affirmer son autorité de seigneur par le rappel de ses enseignements.

Or on trouve les précédents les plus significatifs de dialogues entre un personnage et des personnifications dans la littérature allégorique et didactique, où ces dialogues servent à la révélation et à la transmission d'un enseignement; c'est le cas dans le genre doctrinal et édifiant bien sâr, mais aussi dans le domaine profanc, et 


\section{La Tenson avec le Dieu d' Amour}

il peut suffire de rappeler le Roman de la Rose, et pour l'Italie, le Tesoretto. Dans notre tenson, le Dieu d'Amour se prévaut précisément du statut d'autorité que ces textes conferent aux personnifications. Ses interventions se présentent comme des discours de type didactique ou doctrinal. A cet égard, les apostrophes sont significatives : chaque sonnet contient (généralement au premier vers) l'apostrophe Amore dans les sonnets de l'amant, Amante dans les sonnets d'Amour (procédé qui devait sans doute renforcer la lisibilité de la tenson, ne serait-ce que pour les copistes). Or on observe que pour l'amant prononcer le nom d'Amour a une valeur affective; il l'accuse, il l'implore, etc. Amour par contre utilise tour à tour amante' comme dénomination et comme un nom commun. Dans le sonnet 4 Amour joue sur cette ambiguïté: «'Amante', no, ma 'disamante'dico » (donc: "tu n'as pas mérité le nom d'amant "); dans le sonnet 8 amante est un nom commun; dans le sonnet 10 , un mini-traité de l'art d'aimer entièrement construit sur des mots dérivés de la racine 'aimer'(amanza, amorosa, amar) amante est a la fois l'interlocuteur (v. 5) et le nom commun (v. 2). Amour considère donc l'expérience singulière de l'amant à la lumière d'une doctrine générale sur ce que doit faire le bon amant.

Une visée didactique semble aussi motiver la seule reprise de rimes repérable dans l'ensemble de la tenson: la fronte du sonnet 2 reprend la rime aggio des tercets du sonnet 1 . Or cette rime réapparaît dans le dernier sonnet de l'amant (11). Une analyse des mots à la rime est éclairante: dans le sonnet 1 nous trouvons, à côté de deux verbes au futur (faraggio, morraggio), vassallaggio; dans la réponse d'Amour (2) nous trouvons messaggio, coraggio, aggio, viaggio et usaggio. Or viaggio réapparaît dans le sonnet 11: viaggio, danaggio, saggio, ubideraggio et sengnoraggio (qui fait écho à vassallaggio de 2 ) ${ }^{12}$. Ces reprises 
soulignent le concept du service amoureux comme rapport de loyauté féodale, mais évoquent aussi la métaphore du voyage, présente dans d'innombrables textes à visée morale. On peut y ajouter une reprise de mots, à l'intérieur du vers, qui dans les deux derniers sonnets dédouble la métaphore du voyage: à 11, 5 via sola correspond à 12, 5 via verace ${ }^{12}$.

Or nous avions déjà relevé dans les exemples provençaux le caractère évasif des réponses d'Amour. De même, dans la tenson de Monte Andrea, l'introduction, par le biais de la forme dialogique, d'une dimension didactique dans le contexte ly rique, provoque un décalage; elle nous semble induire un effet de distanciation ironique qui, comme l'a rappelé Claude Perrus, caractérise souvent les formes lyriques dialogiques. Si le Dieu d'Amour apparaît comme l'instance d'un savoir supérieur, il ne délivre pas de véritable leçon. Le seul élément concret est l'interdiction de trahir le secret de l'amour. Par ailleurs son discours reste vague et général, et se borne à rappeler quelques termes de l'éthique courtoise ${ }^{14}$.

La visée doctrinale $d u$ discours $d^{\prime}$ Amour est ainsi vidée de son sens et apparaît avant tout comme une stratégie rhétorique. Amour, seigneur implacable qui exige obéissance à ses lois, est aussi, ici, un rhétoriqueur habile qui sait se dérober. Il détourne le sens du dialogue. L'amant ne lui avait pas demandé de préceptes, ni un manuel du parfait amant, mais lui avait posé une question bien précise: moi, qui suis ton fidèle serviteur, n'ai-je pas droit à une récompense? Amour esquive ce sujet et ramène le discours à la logique de l'amour courtois : c'est l'exercice des vertus qui qualifie l'amant idéal et qui constitue une fin en soi. Mais l'autorité doctrinale d'Amour ainsi que la doctrine de l'amour courtois sortent bicn affaiblies de cette tenson. Comme dans les exemples 


\section{La Tenson avec le Dieu d' Amour}

provençaux évoqués au début, le dialogue $\mathbf{n}^{\prime}$ apporte pas de réponse, mais traduit une crise. Le jeu complexe de connexions et d'échos entre les sonnets cache l'opposition de deux voix qui ne peuvent dialoguer. Les deux interlocuteurs se servent du même langage, des mêmes images et métaphores, et des mêmes artifices rhétoriques, mais leurs voix appartiennent à deux instances, la loi de l'Amour et le désir de l'amant, qu'aucun artifice, ni langagier, ni doctrinal, ne semble pouvoir concilier.

Johannes BARTUSCHAT 


\section{ANNEXE}

Monte Andrea, Rime

Sonnet 85 (=1 de la tenson)

Si je pouvais le montrer à l'extérieur ou le dire ouvertement avec ma langue en cet état où est réduit mon coeur et chacun de mes membres de chair, je sais qu'Amour en éprouverait de la pitié, de sorte qu'il irait trouver l'avenante dame et lui conterait ma mortelle douleur et la grande peine qui $\mathrm{m}^{\prime}$ afflige parce que sa haute valeur me dédaigne et ne condescend en rien à me complaire.

Puisque je ne peux parler, Amour, que vais-je faire?

Car toi seul $\mathrm{m}^{\prime}$ as amené en cette extrémité!

Si tu ne m'aides pas, tu sais bien que je mourrai.

Amour, aie pitié de moi, ne serait-ce qu'un peu: celle à qui tu $\mathrm{m}^{\prime}$ as donné en vasselage, prie-la de me tirer de ce brasier.

Sonnet 86 ( $=2$ de la tenson)

Tu es un amant d'une bien folle audace en me disant d'être ton messager auprès de celle à cause de qui tu dis languir et souffrir dans le cœur un grand tourment! Tu crois donc, fou, que $j$ 'irais dire un mensonge aussi grand que celui que je viens d'entendre, sachant que je connais ta faute et que tu ne suis pas droitement ma voie?

Cela, tu ne peux en rien le cacher ni plus me dissimuler ta conduite mauvaise. 


\section{LA Tenson AVEC le DIEU d’ Amour}

Si tu veux dire que tu es amené à cause de moi à souffrir quelque tourment ou peine, je dis que tu te trompes, car il ne saurait en être ainsi: car un amant auquel je me suis donné et qui ne s'écarte pas de la voie d'amour, toujours son espoir est comblé par la joie. 


\section{JOHANNES BARTUSCHAT}

\section{NOTES}

1. Pour l'Italie l'étude la plus complète (et la seule étude d'ensemble) sur la tenson du treizième siècle reste celle de $\mathrm{H}$. STIEFEL, Die italienische Tenzone des XIII hahrhunderts und ilu. Verhïlthis zur provenzalischen Tenzone, Halle, Niemeyer, 1914. Elle contient d'ailleurs aux pages 71-72 une brève présentation de notre texte.

2. Ce sont les sonnets 85 à 96 de l'édition critique de F. F Minetti (Rime, Florence, Accademia della Crusca, 1979)

3. On peut penser aussi au Fiore, analysable en un certain sens comme une immense couronne de sonnets et qui contient de nombreux échanges dialogiques. Pour des tensons fictives sous forme de sonnets dans la littérature du Dileciento, voir $\mathrm{H}$. STIEFEL, op. cit., p. 66 suiv.

4. M. VUYLSTEKE, Amor dans la litterature pronensule, "Revue belge de philologie et d'histoire ", LIII (1975), p. 805-814.

5. Qunnt Amors trobet partit, dans M. DE RIQUER, Los trozudores. Historia literaria y textos, Barcelone, Plantea, 1975, vol. II, p. 1120 suiv.

6. S. ASPERTI, Il trozatore Raimon Jordan, Modène, Nucchi, 1990, p. 349 suiv.

7. W.P. SHEPARD/F.M. CHAMBERS, The Poems of Aimeri- de Peguilluan, Evanston, Northwestern University Press, 1950, p. 133-136.

8. Cf. dans le sonnet 6 : piacesse, dispiacessie, dismmi e diszrnoli.

9. Sur ce «dédoublement » et, plus en général, sur les différents emplois de la métaphore du service d'amour, notamment chez les poètes siciliens, cf. C. PERRUS, L'amour en personme dans la poésie du XIII" siêcle, "Chroniques Italiennes", 24 (1990), p. 7-15.

10. L'altrier, dormendo, a mi se vemhe Amore dans G. Savino, piccolo canzoniere di Paolo Lminfranchi da Pistoin, "Filologia e critica », VII (1982), p. 68-96, p. 83-84 (sonnet suivi d'une poésie structurée comme dialogue poeta-Amore). 


\section{La Tenson avec le Dieu d’ Amour}

11. Cf. aussi 2,14 .

12. Cf, les rimes viaggio, danaggio, coraggio, sengnoraggio, aggio du sonnet Si come i marinar'guida la stella (n. 33 de l'édition Minetti).

13. Cf. aussi 8, 2 : via amorosa.

14. Notre lecture peut recevoir confirmation d'une autre tenson entre l'amant et Amour, les sonnets 42 à 44 de la Corona di casistica d'amore de l' " amico di Dante "(G. CONTINI (ed.), Poeti del Duecento, Milan-Naples, Ricciardi, 1960, vol. II, p. 760-762). Comme chez Monte, l'échange est motivé par les lamentations de l'amant qui accuse Amour d'indifférence et de manquer à ses obligations vis-à-vis d'un serviteur fidèle. Amour reproche à l'amant son attitude et se borne par ailleurs à l'inviter à suivre ses préceptes (qui sont les préccptes de l'homme courtois) en lui promettant qu'il connaîtra la « joie ". 